Journal of Computer Science 3 (11): 863-868, 2007

ISSN 1549-3636

(C) 2007 Science Publications

\title{
Performance Evaluation of Modified AODV for Load Balancing
}

\author{
${ }^{1}$ Amita Rani and ${ }^{2}$ Mayank Dave \\ ${ }^{1}$ Department of Computer Science and Engineering, UIET, \\ Kurukshetra, HR, India-136119 \\ ${ }^{2}$ Department of Computer Engineering, National Institute of Technology, \\ Kurukshetra, HR, India-136119
}

\begin{abstract}
AODV is a prominent routing protocol for MANET that uses hop count as a path selection metric. However, AODV has no means to convey traffic load on current route. This study focuses on introducing a new metric, Aggregate Interface Queue Length (AIQL), in AODV in order to deal with load balancing issues. Performance evaluation through simulation shows that the modified code can perform better than the conventional AODV. We also evaluate the effect of interface queue length on normalized routing load, average throughput and average end-to-end delay.
\end{abstract}

Key words: Ad hoc networks, aggregate interface queue length, average throughput, normalized routing load, end-to-end delay

\section{INTRODUCTION}

A mobile ad hoc network is defined as a collection of mobile platforms or nodes where each node is free to move about arbitrarily. Each node logically consists of a router that may have multiple hosts and that also may have multiple wireless communication devices ${ }^{[1]}$. The routing protocols in MANETs can be categorized in to three different groups: Global/Proactive, Ondemand/Reactive and Hybrid routing protocols ${ }^{[2]}$. In global routing protocols, each node stores and maintains routing information to every other node in the network. In on-demand routing protocols, routes are created when required by the source node, rather than storing up-to-date routing tables. Hybrid routing protocols combine the basic properties of the two classes of protocols mentioned earlier. In practice, some routes get congested, while other routes remain underutilized. This results in poor performance of mobile ad hoc networks. Therefore, the need for balancing the load distribution among various routes becomes more important.

The aim of this study is to provide a characteristic comparison for a number of proposed load balanced ad hoc routing protoc ols and propose a modification to $\mathrm{Ad}$ hoc On-demand Distance Vector (AODV) routing protocol that balances the load on various routes. We evaluate the effect of varying interface queue lengths on average throughput, normalized routing load and end to end delay. We perform this study using the Ad hoc On demand Distance Vector (AODV) routing protocol, modified AODV and ns-2.29 as the simulation tool.

\section{BACKGROUND OF THE LOAD BALANCING ROUTING ALGORITHMS}

Load balancing deals with improving the performance of the system by transferring the jobs from overloaded nodes to underloaded or idle nodes. By doing so, the total time to process all jobs may reduce considerably and also makes it sure that no node sits idle while some jobs are waiting to be processed. Routing protocols are vital for the proper functioning of ad hoc networks. A major drawback of all existing ad hoc routing protocols is that they do not have provisions for conveying the load and/or quality of a path during route setup. Hence they cannot balance the load on different routes. Also, both proactive and reactive protocols chose a route based on the metric, the smallest number of hops to the destination. But it may not be the most significant route when there is congestion or bottleneck in the network. It may cause the packet drop rate, packet end-to-end delay, or routing overhead to be increased particularly in the cases when the traffic is concentrated on a special node like a gateway through which mobile nodes from ad hoc network can connect to Internet.

There are various proposed algorithms for load balancing that consider traffic load as a route selector, but these algorithms neither reflect burst traffic nor transient congestion ${ }^{[3]}$. In order to ensure uninterrupted communication and in order to make routing protocols more efficient in presence of node movement, two issues, Route maintenance and Bandwidth reservation 
Table 1: Characteristics of load balanced ad hoc routing protocols

\begin{tabular}{|c|c|c|c|c|}
\hline Routing protocol & Metric used & RS & BR & LBE \\
\hline Load Balanced Ad hoc Routing (LBAR) ${ }^{[6]}$ & Degree of nodal activity & $\mathrm{F}$ & $\mathrm{Y}$ & Network \\
\hline Dynamic Load Aware Routing (DLAR) $)^{[7]}$ & Intermediate node routing load & $\mathrm{F}$ & $\mathrm{Y}$ & Network \\
\hline Load Sensitive Routing (LSR) ${ }^{[8]}$ & $\begin{array}{l}\text { Summation of number of queuing packets at } \\
\text { mobile host and at neighboring hosts }\end{array}$ & $\mathrm{F}$ & $\mathrm{N}$ & Network \\
\hline Weighted Load Aware Routing (WLAR) ${ }^{[9]}$ & *Traffic load & $\mathrm{F}$ & $\mathrm{Y}$ & Network \\
\hline $\begin{array}{l}\text { Load Aware Routing in Ad hoc networks } \\
\text { (LARA) }^{[10]}\end{array}$ & $\begin{array}{l}\text { Contention at the MAC level for non-TCP } \\
\text { source } \\
\text { Number of hops and traffic cost of the route } \\
\text { for TCP source }\end{array}$ & $\mathrm{F}$ & $\mathrm{Y}$ & Network \\
\hline Correlated Load-Aware Routing (CLAR) ${ }^{[11]}$ & Traffic load through and around neighboring & $\mathrm{F}$ & $\mathrm{Y}$ & Network \\
\hline $\begin{array}{l}\text { Simple Load-balancing Ad hoc Routing } \\
\text { (SLAR) }^{[12]}\end{array}$ & nodes Forwarding Load & $\mathrm{S}$ & $\mathrm{S}$ & Node \\
\hline Simple Load-balancing Approach (SLA) ${ }^{[13]}$ & Own Traffic Load & $\mathrm{S}$ & $\mathrm{S}$ & Node \\
\hline $\begin{array}{l}\text { A Workload-Based Adaptive Load-Balancing } \\
\text { Technique for MANETs }{ }^{[17]}\end{array}$ & Interface queue occupancy and workload & $\mathrm{S}$ & $\mathrm{S}$ & Node \\
\hline $\begin{array}{l}\text { Multipath Routing Protocol with Load } \\
\text { Balancing (MRP-LB) }{ }^{[14]}\end{array}$ & $\begin{array}{l}\text { Total number of congested packets on } \\
\text { multiple routes }\end{array}$ & $\mathrm{F}$ & $\mathrm{Y}$ & Network \\
\hline $\begin{array}{l}\text { Ad hoc On-demand Distance Vector Routing } \\
\text { with Load Balancing (LB-AODV) }\end{array}$ & Balance Index & $\mathrm{H}$ & $\mathrm{N}$ & Network \\
\hline $\begin{array}{l}\text { Protocol to enhance path reliability and realize } \\
\text { load balancing in MANETs }{ }^{[16]}\end{array}$ & Node-disjoint routes & $\mathrm{F}$ & $\mathrm{Y}$ & Network \\
\hline $\begin{array}{l}\text { Load Balancing and Resource Reservation in } \\
\text { Mobile Ad Hoc Networks }\end{array}$ & Bandwidth & $\mathrm{F}$ & $\mathrm{N}$ & Network \\
\hline $\begin{array}{l}\text { Delay-based Load-Aware On demand Routing } \\
\text { (D-LAOR) protocol }{ }^{[18]}\end{array}$ & Total path delay and hop count & $\mathrm{F}$ & $\mathrm{N}$ & Network \\
\hline $\begin{array}{l}\text { Busy Node Avoidance Routing (BNAR) } \\
\text { protocol }^{[19]}\end{array}$ & $* *$ Busy rate & $\mathrm{F}$ & $\mathrm{N}$ & Network \\
\hline $\begin{array}{l}\text { BNAR_with_Netw-ork Allocation Vector } \\
\text { (BNAR_with_NAV) }\end{array}$ & $* *$ Busy rate & $\mathrm{F}$ & $\mathrm{N}$ & Network \\
\hline 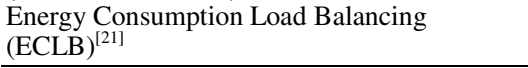 & Energy consumption rate & $\mathrm{F}$ & $\mathrm{N}$ & Network \\
\hline
\end{tabular}

need due mention. A very good solution to these issues is multi path routing. Due to such multi path routing, even if one path fails, data can still be routed to the destination using the other routes. Thus, the cost of rediscovering new path can be salvaged ${ }^{[4]}$. While selecting the path set the following issues need due consideration: ${ }^{[5]}$

- The distribution of load should be even. Mobile nodes with lower traffic load should be preferred to the heavily loaded mobile nodes

- The traffic load in the medium surrounding the mobile nodes on the routes, should be light

- The paths should comprise of nodes with high residual battery power

- If a link is highly reliable, it is advantageous to allow it to be shared by more than one path

A comparative study of some of the load balanced ad hoc routing protocols is in the Table 1 .

\section{A DESCRIPTION OF AODV ROUTING PROTOCOL}

Ad hoc On-demand Distance Vector (AODV) routing protocol is an on-demand protocol that is it discovers routes on an as needed basis using route discovery process. It uses traditional routing tables, one entry per destination to maintain routing information. When a route to a new destination is needed, the node broadcasts a RREQ to find a route to the destination. A route can be determined when the RREQ reaches either the destination itself, or an intermediate node with a 'fresh enough' route to the destination. A 'fresh enough' route is a valid route entry for the destination whose associated sequence number is at least as great as that contained in the RREQ. AODV uses sequence numbers maintained at each destination to determine freshness of routing information and to prevent routing loops. These sequence numbers are carried by all routing packets. The route is made available by unicasting a RREP back to the origination of the RREQ. AODV relies on routing table entries to propagate a RREP back to the source and subsequently, to route data packets to the destination. An important feature of AODV is maintenance of timer-based states in each node, regarding utilization of individual routing table entries. A routing table entry is expired if not used recently. A set of predecessor nodes is maintained for each routing table entry, indicating the set of neighboring nodes that use that entry to route data packets. These nodes are 
notified with RERR packets when the next hop link breaks. Each predecessor node, in turn, forwards the RERR to its own set of predecessors, thus effectively erasing all routes using the broken link.

\section{PROPOSED MODIFICATION TO AODV}

The proposed modification extends AODV and distributes the traffic among ad hoc nodes through a simple load balancing mechanism. The protocol adopts basic AODV procedure.

In this protocol each node measures the number of packets queued up in its interface. Now when a source node initiates a route discovery procedure by flooding RREQ messages, each node receiving an RREQ will rebroadcast it adding its own interface queue length. Destination node will select the best route and replies with RREP.

Route selection procedure: When a source node initiates a route discovery procedure by flooding RREQ messages, each node that receives the RREQ looks in its routing table to see if it has a fresh route to the destination. If it doesn't have the route it adds the number of packets in its interface queue and broadcasts it further. The process is repeated till either the destination is reached or no destination is found.

If an intermediate node has a fresh route to the destination or for the same sequence number the intermediate node has a shorter route or the Aggregate Interface Queue Length (AIQL) is smaller, the intermediate node replies with the route. Here the metric AIQL is the sum of interface queue lengths of all the intermediate nodes from the source node to the current node.

The AIQL metric has been used in order to find out the heavily loaded route. Because if the aggregate queue length for the path is higher, then it obviously means that either all the nodes on the path are loaded or there is at least one node lying on the route that is overloaded. Hence by considering a route with lesser value of aggregate queue length for selecting the path we are diverging the packets from heavily loaded route to comparatively lighter route.

\section{MATERIALS AND METHODS}

In this section we describe our simulation environment and performance metrics.

Simulation environment: We have used ns-2 for our simulations. As mentioned earlier, we have performed our study with AODV and modified AODV protocol.

The AODV protocol in ns-2 maintains a send buffer of 64 packets used in route discovery. The maximum waiting time in the send buffer during route discovery is $30 \mathrm{sec}$. If the packet is in the send buffer for more than $30 \mathrm{sec}$, the packet is dropped.

The default size of the interface buffer of each node is 64 packets, but we have simulated using different values of interface queue length and tried to study the effect of variable sizes of queue length.

We used 50 mobile nodes in a rectangular grid of dimensions $1500 \times 300 \mathrm{~m}$. We run each simulation for $600 \mathrm{sec}$. We used the random waypoint model because it is the most widely used mobility model in previous studies. The maximum allowed speed for a node was $20 \mathrm{~m} \mathrm{sec}^{-1}$.

We used a Constant Bit Rate (CBR) source as the data source for each node. Each source node transmitted packets at the rate of four packets per sec, with a packet size of 512 bytes. We varied the traffic load, the degree of mobility and the size of interface queue length in the simulations. We have varied the traffic load by varying the number of sources to be 10 , 20, 30 and 40. We control the degree of mobility by varying the pause times as $0,30,60,120,300$ and $600 \mathrm{sec}$. A movement scenario arranges the movement and the position of the nodes according to the random waypoint model.

Performance metrics: We use the following performance metric to evaluate the effect of each scheduling algorithm:

Normalized routing load: The number of routing control packets per data packet delivered at the destination.

Average end-to-end delay: This is the average overall delay for a packet to traverse from a source node to a destination node. This includes the route discovery time, the queuing delay at a node, the transmission delay at the MAC layer and the propagation and transfer time in the wireless channel.

Average throughput: It is defined as the ratio of total packets received to the simulation time.

\section{EVALUATION OF ROUTING ALGORITHMS}

The important feature of our study is that we have performed simulations from an entirely new perspective and as per our knowledge no such study has been done before. To evaluate the routing algorithms, we first look at the effect of interface queue length on Average throughput and then provide some reasons for the behavior. We then study the impact of interface queue 
length on normalized routing load and end-to-end delay for AODV and the proposed modification. We have studied the above mentioned performance metrics by varying the number of sources at different pause times. Due to space constraints we only show the result for pause times of 30 i.e. constantly changing topology and 300 i.e. moderately changing topologies and number of source nodes as 20 and 30 . Simulation for other values show appropriate results.

From Fig. 1-4 it is clear that with the lesser number of sources and highly dynamic topologies the modified AODV protocol gives much better performance than the AODV protocol. Topologies are highly dynamic when pause time is less or the nodes are continuously moving. As mobility is the basic feature of ad hoc networks, therefore such a simulation behavior of modified AODV protocol is a benefit over conventional AODV. As the number of sources increases both the protocols have almost similar behavior. The reason is that with the increase in offered load almost all of the routes get equally loaded and hence the value of AIQL is almost same for all routes.

We also observe that for highly dynamic topologies the normalized routing load is nearly constant for all values of interface queue length. But for more stable topologies the graphs we get have a curve like structure, i.e. initially the normalized routing load is high, but it decreases as the interface queue length increases till a certain value of interface queue length is reached and again starts increasing with the increase in interface queue length. This means that from this statistics we can get the optimal interface queue length that will give the best performance and can reduce the routing load to a considerable amount.

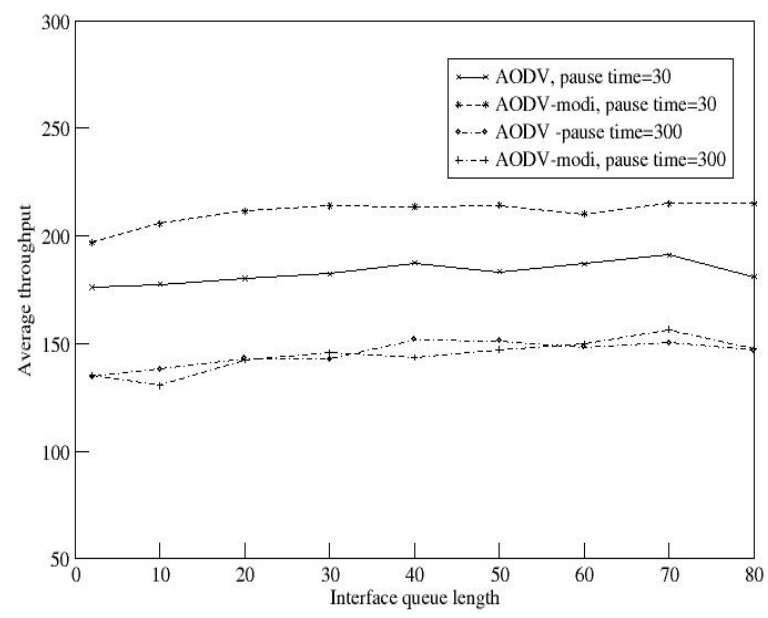

Fig. 1: Average throughput for 20 sources

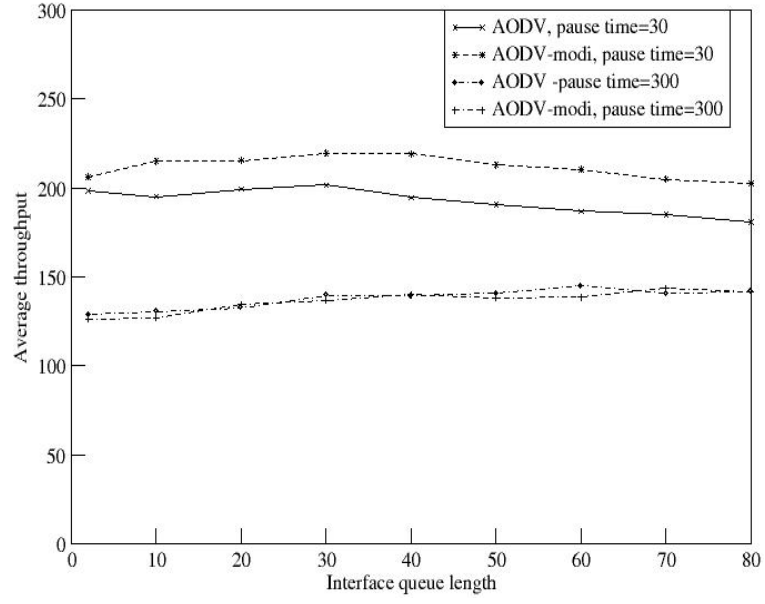

Fig. 2: Average throughput for 30 sources

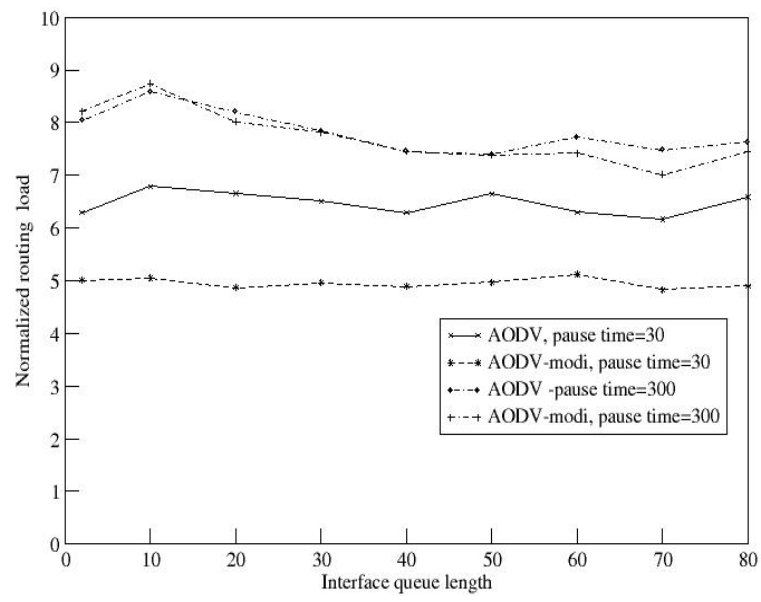

Fig. 3: Normalized routing load for 20 sources

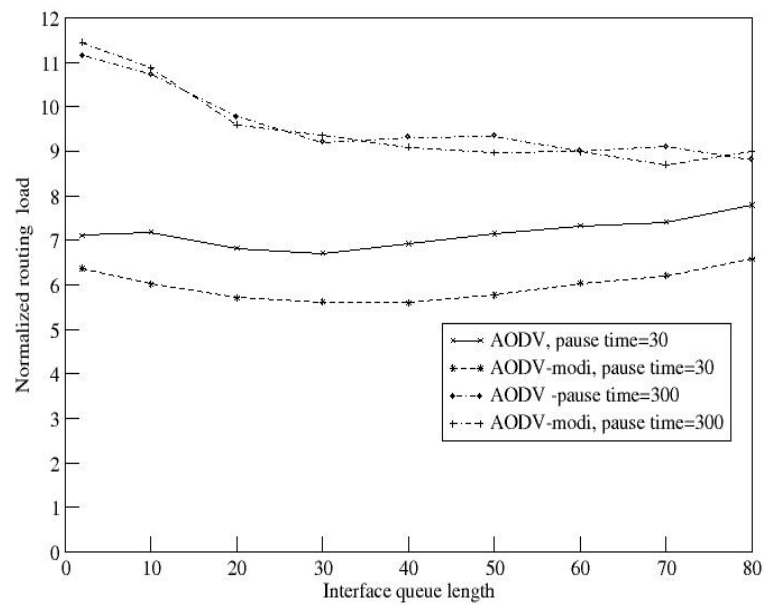

Fig. 4: Normalized routing load for 30 sources 


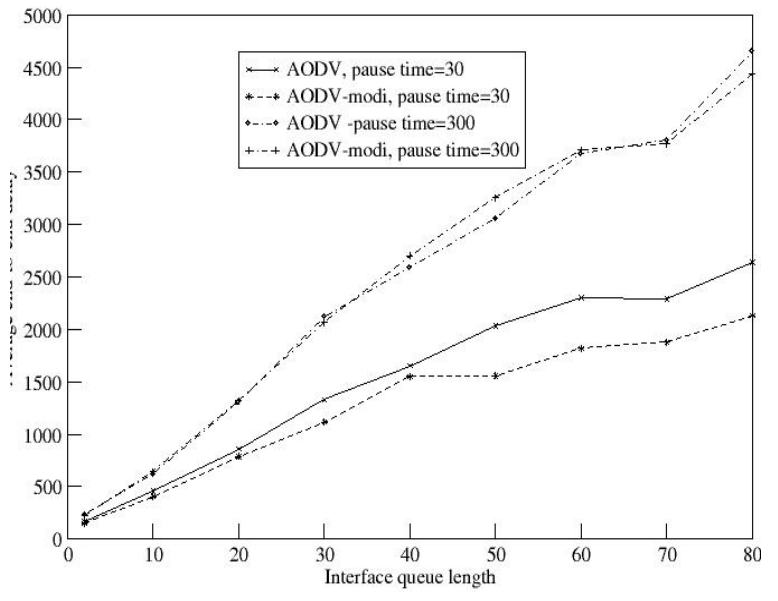

Fig. 5: Average end-to-end delay for 20 sources

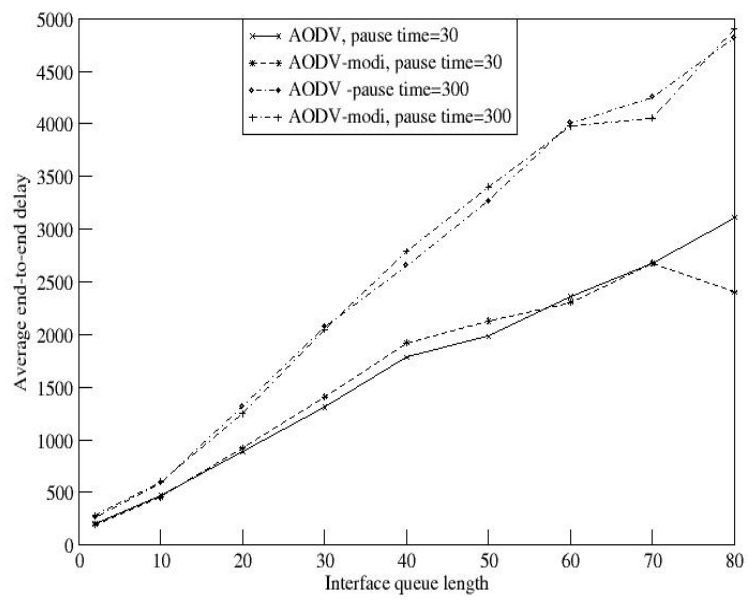

Fig. 6: Average end-to-end delay for 30 sources

We next observe that as expected average end-toend delay increases with the increase in buffer size. The effect of buffer size is not that prominent for performance metrics like average throughput and normalized routing load as is for average end-to-end delay. Average end-to-end delay is lowest for smallest value of buffer size. The reason is quite obvious, packets do not have to wait for long in the queue and hence they reach early resulting in low value of end-toend delay. However, very small size of buffer leads to large number of packets dropped. Packets are dropped because small buffers fill early and the packets arriving after buffer fill are dropped (Fig. 5, 6).

\section{CONCLUSION}

In this study we proposed a modification to AODV for distributing traffic load among nodes in ad hoc networks. The key concept of modification is to provide a metric for load distribution, the selection of light load path and the reduction of congested nodes in high load networks. We performed a simulation study and compared the modified version of AODV with traditional AODV protocol. The simulation results show that the modifications can improve average throughput and reduce normalized routing load by keeping track of the aggregate interface queue length. However, the modifications to AODV are more useful to moderately loaded high mobility networks.

\section{REFERENCES}

1. Perkins, C.E., 2001. Ad Hoc Networking: Addison Wesley, USA.

2. Sun, J.Z. and J. Sauvola, 2002. On Fundamental Concept of Mobility for Mobile Communications: Proc. Of 13th IEEE International Symposium on Personal, Indoor and Mobile Radio Communication, Lisbon, Portugal, 2: 799-803.

3. Abolhasan, M., T. Wysocki and E. Dutkiewicz, 2001. A Review of Current On-demand Routing Protocols: ICN 2001, LNCS 2094, pp: 186-195.

4. Perkins, C.E. and P. Bhagwat, 1994. Highly Dynamic Destination Sequenced Distance Vector Routing for Mobile Computers: Proc. Of Computer Communication Rev., 1: 234-244.

5. Murthy, S. and J.J. Garcia-Luna-Aceves, 1996. An Efficient Routing Protocol for Wireless Networks: ACM/Baltzer Mobile Networks and Applications, 1 (2): 183-197.

6. Hassanein, H. and A. Zhou, 2001. Routing with Load Balancing in Wireless Ad Hoc Networks: Proc. ACM MSWiM, Rome, Italy, pp: 89-96.

7. Lee, S.J. and M. Gerla, 2001. Dynamic Load Aware Routing in Ad Hoc Networks: Proc. ICC 2001, Helinski, Finland, pp: 3206-3210.

8. Wu, K. and J. Harms, 2001. Load Sensitive Routing for Mobile Ad Hoc Networks: Proc. IEEE ICCCN'01, Phoenix, AZ, pp: 540-546.

9. Choi, D.I., J.W. Jung, K.Y. Kwon, D. Montgomery and H.K. Kahng, 2003. Design and Simulation Result of a Weighted Aware Routing (WLAR) Protocol in Mobile Ad Hoc Network: LNCS 3391, pp: 178-187.

10. Saigal, V., A.K. Nayak, S.K. Pradhan and R. Mall, 2004. Load Balanced Routing in Mobile Ad hoc Networks: Elsevier Computer Communications 27, pp: 295-305.

11. J.W. Jung, D.I. Choi, K. Kwon, I. Chong, K. Lim and H.K. Kahng, 2004. A Correlated Load Aware Routing Protocol in Mobile Ad Hoc Networks: ECUMN 2004, LNCS 3262, pp: 227-236. 
12. Ahn, S., Y. Lim and J. Choe, 2003. A LoadBalancing Approach in Ad-Hoc Networks: ICOIN 2003, LNCS 2662, pp: 672-681.

13. Yoo, Y. and S. Ahn, 2004. A Simple LoadBalancing Approach in Secure Ad Hoc Networks: ICOIN 2004, LNCS 3090, pp: 44-53.

14. Pham, P.P. and S. Perreau, 2004. Increasing the network performance using multi-path routing mechanism with load balance: Ad Hoc Networks, 2 (4): 433-459.

15. J.H. Song, V.W.S. Wong and V.C.M. Leung, 2003. Efficient On-Demand Routing for Mobile Ad-Hoc Wireless Access Networks: IEEE GLOBECOM 2003, pp: 558-563.

16. Argyriou and V. Madisetti, 2006. Using a new protocol to enhance path reliability and realize load balancing in mobile ad hoc networks: Elsevier Ad Hoc Networks 4, pp: 60-74.

17. Lee, Y.J. and G.F. Riley, 2005. A Workload-Based Adaptive Load-Balancing Technique for Mobile Ad Hoc Networks: IEEE Communication Society, WCNC 2005, pp: 2002-2007.
18. Song, J.H., V. Wong and V.C.M. Leung, 2003. Load-Aware On-Demand Routing (LAOR) Protocol for Mobile Ad hoc Networks: The 57th IEEE Semiannual Vehicular Technology Conference, p3: 1753-1757.

19. Hakoda, J., H. Uehara and M. Yokoyama, 2002. Performance Evaluation of Mobile Ad Hoc Routing Protocols Based on Link Expiration Time and Load of Node: IEICE Trans. Commun., J85B (12): 2108-2118.

20. Takahashi, S., J. Hakoda, H. Uehara and M. Yokoyama, 2004. A Load Balanced Routing Scheme for Mobile Ad Hoc Networks: ISITA2004, Parma, Italy.

21. Cho, H.K., E.S. Kim and D.W. Kang, 2005. A Load-balancing Routing Considering Power Conservation in Wireless Ad-Hoc Networks: Proc. Of the 16th International Workshop on Database and Expert Systems Applications, DEXA'05.

22. Chakrabati, G. and S. Kulkarni, 2004. Load Balancing and Resource Reservation in Mobile Ad hoc networks: Elsevier Ad Hoc Networks, accepted April, 2004. 